\title{
Simulated Ion Trajectory and Induced Signal in Ion Cyclotron Resonance Ion Traps
}

\author{
Xinzhen Xiang \\ Lepartment of Chemistry, The Ohio State University, Columbus, Ohio, USA \\ Shenheng Guan and Alan G. Marshall \\ National High Magnetic Field Laboratory and Department of Chemistry, Florida State University, Tallahassee, \\ Florida, USA
}

\begin{abstract}
We present a numerical method for computation of electrostatic (trapping) and time-varying (excitation) electric fields and the resulting ion trajectory and detected time-domain-induced voltage signal in a rectangular (or cubic) ion cyclotron resonance (ICR) ion trap. The electric potential is calculated by use of the superposition principle and relaxation method with a large number of grid points (e.g., $100 \times 100 \times 100$ for a cubic trap). Complex ICR experiments and spectra may now be simulated with high accuracy. Ion trajectories may be obtained for any combination of trapping and excitation modes, including quadrupolar or cubic trapping in static or dynamic mode; and dipolar, quadrupolar, or parametric excitation with single-frequency, frequency-sweep (chirp), or stored waveform inverse Fourier transform waveforms. The resulting ion trajectory may be represented either as its threedimensional spatial path or as two-dimensional plots of $x-, y-$, or z-position, velocity, or kinetic energy versus time in the absence or presence of excitation. Induced current is calculated by use of the reciprocity principle, and simulated ICR mass spectra are generated by Fourier transform of the corresponding time-domain voltage signal. (J Am Soc Mass Spectrom 1994, 5, 238-249)
\end{abstract}

$\mathrm{F}$ Tourier transform ion cyclotron resonance mass spectrometry (FT/ICR/MS) has become one of the most promising methods for mass analysis since its introduction more than 19 years ago [1]. Beginning as a technique for the study of gas-phase ion-molecule reactions, FT/ICR/MS has emerged as a powerful analytical tool capable of ultrahigh mass resolving power, multistage tandem mass spectrometry (MS/MS), wide mass range, simultaneous detection of all ions, and long ion storage periods [2-23]. Full realization of these capabilities requires deep understanding of various means and mechanisms for trapping, excitation, and detection of ions in FT/ICR traps.

Ion motions in a quadrupolar electrostatic trapping potential. An FT/ICR/MS experiment is carried out in an ICR ion trap, which has three functions: (1) to confine ions for collisions, chemical reactions, photo-induced processes, etc.; (2) excitation and/or ejection of massselected trapped ions; and (3) detection of massselected trapped ions. Ions generated in or injected into an ICR ion trap are usually held there by the combination of a strong static magnetic field and an electrostatic trapping potential. Generation of a static electric

Address reprint requests to Prof. Alan G. Marshall, National High Magnetic Field Laboratory, Florida State University, 1800 East Paul Dirac Drive, Tallahassee, FL 32306 4005. potential minimum (at the center of the trap) as a function of the "axial" dimension (i.e., along the magnetic field direction) must result in an electric potential maximum (at the center of the trap) as a function of the other (lateral or radial) dimension. The simplest trapping potential is quadrupolar, that is, it varies quadratically along either the axial or radial direction (see Theory). A near-perfect quadrupolar trapping potential may be generated by a trap consisting of a ring and two endcap electrodes with hyperbolic shapes. Such "Penning" traps are widely used by the physics community for studies of single trapped ions [24-30]. In a Penning trap, the quadrupolar electrostatic potential varies quadratically along the axial (z-) direction, with a potential minimum located at the center of the trap. In any (xy-) plane perpendicular to the magnetic field, the trapping potential is always maximal along a line between the centers of the two endcaps. Although ion motion in the xy-plane is thus not stable, ions remain trapped on an equipotential contour if no energy loss mechanism (e.g., ion-neutral collisions) is available. In an infinitely extended perfectly shaped empty hyperbolic trap, the trapping potential is parabolic and the equipotential contours of the electrostatic trapping potential in a given $x y$-plane are perfectly circular (see Results and Discussion). The equations describing ion motions in the absence of any excitation electric field in such a Penning trap may be 
solved analytically [24]. Ion axial and radial motions are independent: ions oscillate harmonically along the magnetic field (axial) direction and rotate at magnetron and/or cyclotron frequencies about a guiding line parallel to the magnetic field direction. The ion cyclotron frequency is independent of ion position in a purely quadrupolar trapping potential. Two-dimensional ion motion in a spatially uniform electric excitation field and in the absence of an electrostatic trapping potential has been analyzed [31] for frequencysweep $[32,33]$ and stored waveform inverse Fourier transform (SWIFT) [34] excitation.

Nonquadrupolar traps. For ICR experiments involving ions of more than one mass-to-charge ratio, the hyperbolic trap has several disadvantages. First, trap construction is difficult. Second, the necessary truncation of the trap to finite size and the placement of holes in the endcaps to provide for introduction of ionizing beam(s) warps the electrostatic potential, leading to various (generally undesirable) nonlinearities (see below). Such distortions are less serious in single-ion experiments, which are generally conducted at much smaller ion cyclotron radius than FT/ICR experiments based on image-charge detection $[35,36]$. Third, the hyperbolic electrodes protrude into the trap, limiting the number of ions which may be trapped at a given charge density. Fourth, although the hyperbolic electrodes are optimal for ion trapping, they are quite nonoptimal for excitation and detection, for which a spatially uniform (rather than quadrupolar) electric field is needed. Attempts to produce dipolar excitation and detection with a segmented Penning-type trap result in increased multiple-harmonic frequency signals [37] and z-ejection [38]. The same problem (although less serious) is true of a segmented cylindrical trap $[39,40]$. Although more complex designs with better trapping and radiofrequency (RF) performance are available [41-44], the widely used cubic trap [45] offers a good compromise for approximately quadrupolar trapping potential and approximately uniform azimuthal dipolar excitation.

For an actual ICR ion trap, a solution of Laplace's equation for the electrical potential in an empty rectangular [46, 47] or cylindrical trap [48] may be obtained from an infinite series expansion of the potential about the center of the trap. For ions near the center of the trap, the trapping potential may be approximated as a quadrupolar potential. Thus, in the absence of an (time-varying) excitatory electric field, inn trajectories near the center of the trap may be analyzed into the three independent cyclotron, magnetron, and axial trapping oscillation motions noted above.

Away from the center of the trap the potential deviates from the quadrupolar approximation, and higher-order potential terms must be included. In a nonquadrupolar electrostatic trapping potential field, the cyclotron, magnetron, and axial frequencies all depend on ion spatial position. For example, Dunbar et al. [49] have demonstrated theoretically that magnetron frequency decreases as ions move outward from the center of the trap.

Time-varying electric excitation potential in an ICR ion trap. To a first approximation, second or higher-order terms in the excitation electric potential may be neglected (i.e., the excitation electric field is taken as spatially uniform - linearized and RF-shimmed $[42,50]$ traps approximate this condition) and the trapping potential may be assumed to be quadrupolar. At this level of approximation, ion motion obeys a linear differential equation which may be solved analytically $[24,33,51,52]$. Response of ion motions to more complicated excitation waveforms may be understood by means of linear response theory, which relates, for example, the postexcitation ion cyclotron orbital radius to the Fourier components at the ICR frequency of the excitation field $[53,54]$.

Experimentally, nonlinear behavior of ions during excitation is observed in either a cubic or cylindrical trap, for example, variation in detected relative ion abundances at different rates of frequency-sweep excitation $[42,55-60]$. Ion ejection by dipolar azimuthal (radial) excitation to a cyclotron radius equal to the radius of the trap also becomes nonlinear in a spatially nonuniform electric excitation field. In an attempt to describe such nonlinear behavior, equations with terms up to fourth order in the excitation potential have been solved, and mechanisms of coupling between the radial motion and $z$-oscillation have been proposed [40, 61]. In a relaled approach, Mitchell [62] has developed a method in which, for example, nonquadrupolar electrostatic trapping potential terms are treated as a perturbation of the quadrupolar potential in the spirit of quantum mechanical perturbation theory. A problem common to such analytical treatments is that both the electrostatic trapping potential and the electric excitation potential are approximated by truncating the power series spatial expansion of either potential to a small number of terms; the effect of the truncation is thus not known.

Alternatively, a numerical method based on an infinite series expansion of the potential has been used to solve the equations of motion for excitation at twice the axial oscillation frequency, $2 \omega_{\mathrm{z}}[59]$. However, that treatment employed a spatial grid of only $10 \times 10 \times 10$ points, which did not provide sufficient accuracy for comparison to experimental observations.

Present approach. In this article, we present and develop a numerical method to compute the electrostatic trapping potential in an orthorhombic ICR ion trap, from which we may solve for an ion trajectory in the absence or presence of time-varying electric excitation potential (also evaluated numerically). From the ion trajectory we then compute the induced differential charge (whose time-dependence gives a differential 
current which may be converted into a voltage signal, whose Fourier transform yields the FT/ICR spectrum) between a pair of opposed detection electrodes. Because of the symmetry of a cubic trap, it is convenient to calculate an "eigen" potential and construct an arbitrary potential at any given time from the eigenpotential by use of the superposition principle (see Theory). The eigenpotential is constructed by a numerical relaxation method for a three-dimensional $100 \times 100$ $\times 100$ grid. Spatial resolution is further enhanced by interpolation. Ion trajectories may then be simulated for a variety of ion initial positions and motional amplitudes for various excitation modes. Effects of relativistic shift [63] and magnetic field inhomogeneity are not included in this article. The method developed in this article provides an accurate and general description of ion trajectories and response to excitation and ejection in an actual cubic ICR ion trap.

\section{Theory}

Static and time-varying electric potentials in a cubic ICR ion trap. The detected ICR signal may be evaluated directly from the ion trajectory, which is in turn calculated from the instantaneous force acting on that ion. The electric component of the Lorentz force (see below) is in turn proportional to the electric field at that point in space, and the electric field is obtained as the gradient of the electric potential. Thus, any attempt to determine the ICR signal response to a given combination of static and time-varying forces must begin from knowledge of the electric potential at any interior location in the ICR ion trap (e.g., a cubic trap). At any instant, $t$, the electric potential, $\Phi(x, y, z, t)$, at each boundary of a cubic ion trap of edge length, $a$, may be expressed as

$$
\begin{aligned}
& \Phi(x, y, z, t)=V_{x+}(t), \\
& x=+a / 2,-a / 2 \leq y \leq a / 2,-a / 2 \leq z \leq a / 2 \\
& \Phi(x, y, z, t)=V_{x-}(t), \\
& x=-a / 2,-a / 2 \leq y \leq a / 2,-a / 2 \leq z \leq a / 2 \\
& \Phi(x, y, z, t)=V_{y^{+}}(t) \\
& \quad y=+a / 2,-a / 2 \leq x \leq a / 2,-a / 2 \leq z \leq a / 2 \\
& \Phi(x, y, z, t)=V_{y-}(t), \\
& \quad y=-a / 2,-a / 2 \leq x \leq a / 2,-a / 2 \leq z \leq a / 2 \\
& \Phi(x, y, z, t)=V_{z+}(t), \\
& z=+a / 2,-a / 2 \leq x \leq a / 2,-a / 2 \leq y \leq a / 2 \\
& \Phi(x, y, z, t)=V_{z-}(t), \\
& z=-a / 2,-a / 2 \leq x \leq a / 2,-a / 2 \leq y \leq a / 2
\end{aligned}
$$

The net potential acting on an ion inside an ICR ion trap is the sum of the potential due to voltage(s) applied to the six boundary electrodes and the spacecharge potential from other ions inside the trap (i.e., the electric potential generated by the ions themselves).
In typical experiments the effect of space charge of other ions is relatively small compared to that from the potential applied to the trap. Therefore, space-charge effects are neglected in the present treatment. The potential inside the trap at time, $t$, due to voltages applied to the boundary electrodes may be obtained by solving Laplace's equation subject to the boundary conditions of eq 1

$\nabla \Phi(x, y, z, t)$

$$
\begin{aligned}
= & \left(\frac{\partial^{2}}{\partial x^{2}}+\frac{\partial^{2}}{\partial y^{2}}+\frac{\partial^{2}}{\partial z^{2}}\right) \Phi(x, y, z, t)=0 \\
& -a / 2 \leq x \leq a / 2,-a / 2 \leq y \leq a / 2, \\
& -a / 2 \leq z \leq a / 2
\end{aligned}
$$

It is in yeneral not practicial to solve ey 2 at each instant during a given excitation or observation event. However, because the Laplace equation is a linear differential equation, we may solve it for a particular boundary condition, and then simply rescale the result for any other boundary condition. Specifically, suppose we apply $1 \mathrm{~V}$ only to the electrode defined by $x=$ $-\mathfrak{a} / 2$

$$
\begin{aligned}
\Phi_{0}(x, y, z) & =1, \\
x & =-a / 2,-a / 2 \leq y \leq a / 2,-a / 2 \leq z \leq a / 2 \\
\Phi_{0}(x, y, z) & =0, \\
x & =a / 2, y= \pm a / 2 \text {, or } z= \pm a / 2
\end{aligned}
$$

Based on the symmetry of a cubic trap, we may express the potential for the whole trap as a superposition of the potentials obtained by applying $1 \mathrm{~V}$ to each plate [e.g., $\Phi_{0}(x, y, z)$ ], each multiplied by the actual voltage applied to that plate:

$$
\begin{aligned}
& \Phi(x, y, z, t) \\
&= V_{x+}(t) \Phi_{0}(x, y, z)+V_{x-}(t) \Phi_{0}(-x, y, z) \\
&+V_{y+}(t) \Phi_{0}(y, x, z)+V_{y_{-}}(t) \Phi_{0}(-y, x, z) \\
&+V_{z+}(t) \Phi_{0}(z, y, x)+V_{z_{-}}(t) \Phi_{0}(-z, y, x)
\end{aligned}
$$

Table 1 lists boundary conditions (i.e., choices of $V_{x+1}$ $V_{x_{-}}$, etc.) corresponding to various possible static and dynamic trapping modes as well as various excitation modes for most ICR experiments. For example, the total potential, $\Phi(x, y, z, t)$, during a radial dipolar excitation process in which a static voltage, $V_{T}$, is applied to each trapping electrode and a time-varying voltage, $V_{x}(t)$, is applied differentially between the x-electrodes (e.g., $V_{x+}=V_{\text {excite }} \sin \omega_{+} l ; V_{x-}=-V_{\text {excite }}$ $\sin \omega_{+} t$ for excitation of cyclotron motion) may be written as

$$
\begin{aligned}
& \Phi(x, y, z, t) \\
& =V_{\text {excite }}(t) \Phi_{0}(x, y, z)-V_{\text {excite }}(t) \Phi_{0}(-x, y, z) \\
& \quad+V_{T} \Phi_{0}(z, y, x)+V_{T} \Phi_{0}(-z, y, x)
\end{aligned}
$$


Table 1. Boundary conditions for various trapping and excitation modes

\begin{tabular}{|c|c|c|c|c|c|c|}
\hline Experimental mode & $x+$ & $x-$ & $y+$ & $y-$ & $z+$ & $z-$ \\
\hline Normal trapping & 0 & 0 & 0 & 0 & $\overline{V_{T}}$ & $\overline{V_{r}}$ \\
\hline Dynamic trapping & 0 & 0 & 0 & 0 & $V_{z} \sin \Omega t$ & $V_{z} \sin \Omega t$ \\
\hline $\begin{array}{l}\text { Azimuthal linear } \\
\text { dipolar cyclotron }\end{array}$ & $V_{x} \sin \omega_{+} t$ & $V_{x} \sin \omega_{+} t$ & 0 & 0 & $v_{T}$ & $v_{T}$ \\
\hline $\begin{array}{l}\text { Azimuthal linear } \\
\text { dipolar magnetron }\end{array}$ & $V_{x} \sin \omega_{-} t$ & $-V_{x} \sin \omega_{-} t$ & 0 & 0 & $V_{T}$ & $v_{r}$ \\
\hline $\begin{array}{l}\text { Azimuthal circular } \\
\text { quadrupolar cyclotron }\end{array}$ & $V_{x} \sin \omega_{+} t$ & $-V_{x} \sin \omega_{+} t$ & $V_{x} \cos \omega_{+} t$ & $-V_{x} \cos \omega_{+} t$ & $v_{T}$ & $V_{T}$ \\
\hline Axial dipolar z-motion & 0 & 0 & 0 & 0 & $V_{r}+V_{2} \sin \omega_{2} t$ & $V_{T}-V_{z} \sin \omega_{z} t$ \\
\hline Azimuthal parametric & $V_{x} \sin \omega_{\rho} t$ & $V_{x} \sin \omega_{p} t$ & $V_{x} \sin \omega_{p} t$ & $V_{x} \sin \omega_{0} t$ & $V_{T}$ & $V_{r}$ \\
\hline Axial parametric & 0 & 0 & 0 & 0 & $V_{T}+V_{2} \sin 2 \omega_{z} t$ & $V_{T}+V_{z} \sin 2 \omega_{z} t$ \\
\hline $\begin{array}{l}\text { Azimuthal quadrupoler } \\
\omega_{+} / \omega_{-} \text {conversion }\end{array}$ & $V_{x} \sin \omega_{c} t$ & $V_{x} \sin \omega_{c} t$ & $V_{x} \sin \omega_{c} t$ & $V_{x} \sin \omega_{c} t$ & $v_{T}$ & $V_{T}$ \\
\hline $\begin{array}{l}\text { Azimuthal quadrupolar } \\
2 \omega_{+} \text {excitation }\end{array}$ & $V_{x} \sin 2 \omega_{+} t$ & $V_{x} \sin 2 \omega_{+} t$ & $-V_{x} \sin 2 \omega_{+} t$ & $-V_{x} \sin 2 \omega_{+} t$ & $V_{T}$ & $V_{T}$ \\
\hline $\begin{array}{l}\text { Azimuthal quadrupolar } \\
2 \omega_{\ldots} \text { excitation }\end{array}$ & $V_{x} \sin 2 \omega_{-} t$ & $V_{x} \sin 2 \omega_{-} t$ & $-v_{x} \sin 2 \omega_{-} t$ & $-V_{x} \sin 2 \omega_{-} t$ & $V_{I}$ & $v_{T}$ \\
\hline
\end{tabular}

The boundary problem of eq 3 may be further simplified by use of the following symmetry-based relations.

$$
\begin{aligned}
\Phi_{0}(x, y, z) & =\Phi_{0}(x,-y, z) \\
& =\Phi_{0}(x, y,-z)=\Phi_{0}(x,-y,-z)
\end{aligned}
$$

Therefore, only the potential within the region, $-a / 2$ $\leq x \leq a / 2,0 \leq y \leq a / 2$, and $0 \leq z \leq a / 2$ need be considered; the potential in any of the three other quadrants may then be obtained from eq 6 . Once the total potential, $\Phi(x, y, z, 0)$, has been calculated for a given ion trap geometry, the data may be stored and simply rescaled to obtain the total potential at any given subsequent time.

Ion trajectory. The motion of a single ion of mass, $m$, and charge, $q$, located at $\mathbf{r}=x \mathbf{i}+y \mathbf{j}+z \mathbf{k}$, with velocity, $\mathbf{v}=\dot{x} \mathbf{i}+\dot{y} \mathbf{j}+\dot{z} \mathbf{k}$, in a magnetic field, $\mathbf{B}$, and electric field, $\mathbf{E}$, is described by the modified Lorentz vector equation [51],

$$
\text { Force }=m \ddot{\mathbf{r}}=q(\mathrm{E}(\mathbf{r}, t)+\dot{\mathbf{r}} \times \mathbf{B}(\mathbf{r}, t))-m \gamma \dot{\mathbf{r}}
$$

which may be expanded in terms of its $x=, y=$, and z-components

$$
\begin{aligned}
& m \dot{x}=q\left[E_{x}(\mathbf{r}, t)+\dot{z} B_{y}(\mathbf{r}, t)-\dot{y} B_{z}(\mathbf{r}, t)\right]-m \gamma \dot{x} \\
& m \dot{y}=q\left[E_{y}(\mathbf{r}, t)+\dot{x} B_{z}(\mathbf{r}, t)-\dot{z} B_{x}(\mathbf{r}, t)\right]-m \gamma \dot{y} \\
& m \dot{z}=q\left[E_{z}(\mathbf{r}, t)+\dot{y} B_{x}(\mathbf{r}, t)-\dot{x} B_{y}(\mathbf{r}, t)\right]-m \gamma \dot{z}
\end{aligned}
$$

in which collisions are represented by a frictional damping force proportional to ion velucity; the frictional coefficient, $y$, represents the reduced collision frequency (i.e., product of the collision frequency and the reduced mass) for momentum transfer [64]. In most FT/ICR/MS experiments, $B(r, t)$ is static and spatially homogeneous, and its direction may be used to define the (negative) z-direction: $B_{z}(\mathbf{r}, t) \mathbf{k}=-B_{0} \mathbf{k}$; $B_{x}(\mathbf{r}, t)=B_{y}(\mathbf{r}, t)=0$.

The electric field, $\mathbf{E}(\mathbf{r}, t)$, is the superposition (i.e., vector sum) of the trapping and excitation fields (see eqs 1) and is obtained as the (negative) gradient of the electric potential, $\Phi(r, t)$

$$
\begin{aligned}
\mathrm{E}(\mathbf{r}, t) & =E_{x}(\mathbf{r}, t) \mathbf{i}+E_{y}(\mathbf{r}, t) \mathbf{j}+E_{z}(\mathbf{r}, t) \mathbf{k} \\
& =-\nabla \Phi(\mathbf{r}, t)
\end{aligned}
$$

or

$$
\begin{aligned}
& E_{\mathbf{x}}(\mathbf{r}, t)=\frac{\partial}{\partial x} \boldsymbol{\Phi}(\mathbf{r}, t) \\
& E_{y}(\mathbf{r}, t)=\frac{\partial}{\partial y} \boldsymbol{\Phi}(\mathbf{r}, t) \\
& E_{z}(\mathbf{r}, t)=\frac{\partial}{\partial z} \Phi(\mathbf{r}, t)
\end{aligned}
$$

Once the time-varying excitation voltages are specified, the electric field may be calculated from eq 4 and the equations of ion motion (eq 7 or eq 8) may be solved for a given choice of ion initial position, $\mathbf{r}(t=0)$, and velocity, $\dot{\mathbf{r}}(t=0)$.

Current induced on detector electrode(s). The current ( $i$ ) induced on each electrode by the ion can be obtained from the reciprocity principle, namely that "the charge induced on an electrode by a unit point charge is equal in magnitude to the potential that would be produced at that same ion position, in the absence of the ion, when a unit potential is applied to the conductive 
electrode $^{\prime \prime}[65-67]$

$$
\frac{Q_{\text {induced }}}{q}=-\frac{V(r)}{V_{0}}
$$

in which $Q_{\text {induced }}$ is the total charge induced on a given electrode by an ion of charge, $q$, located at position $r$; and $V(r)$ is the potential at $r$ when the voltage, $V_{0}$, is applied to the same electrode in the absence of any ions. Thus, the current, $i(t)$, induced on that electrode by a moving ion whose instantaneous position and velocity are $\mathbf{r}$ and $\dot{r}$ is obtained from the time-derivative of $Q_{\text {induced }}$ :

$$
i(t)=q \frac{\dot{\mathbf{r}} \cdot \mathbf{E}(\mathbf{r}, t)}{V_{0}}
$$

in which $q$ is the charge of the ion and $\mathbf{E}(\mathbf{r}, t) / V_{0}$ is the electric field at location, $r$, per unit voltage applied to the detecting electrode. The experimentally detected current at time, $t$, is the sum of the induced currents on both detection electrodes at $y= \pm a / 2$

$$
i(t)=i(t)_{y=a / 2}+i(t)_{y=-a / 2}
$$

The detection equivalent circuit is generally used to model the detected voltage signal before the preamplifier, in which $R$ represents the equivalent resistance of the circuit, and $C$ represents the equivalent caparitance from the pair of opposed parallel receiver plates as well as the cables $[35,36]$. Based on this model, the voltage, $V(t)$, before the preamplifier is then calculated from the following differential equation

$$
C \frac{d V(t)}{d t}+\frac{V(t)}{R}=i(t)
$$

Finally, the frequency-domain ICR spectrum is obtained by taking the (discrete equivalent of the) Fourier transform of the time-domain voltage signal.

$$
F(\omega)=\int_{-\infty}^{+\infty} V(t) \exp ^{-i \omega t} d t
$$

Here we display the "magnitude" or "absolute-value" frequency-domain spectrum, $|F(\omega)|$. Note that the signal components at harmonic frequencies (e.g., $3 \omega_{+}$) are less prominent in the limit of predominantly capacitive detection because in that limit the detected current signal is converted to a voltage signal by dividing by frequency (i.e., the relative magnitude of the signal at third harmonic frequency is one third as large for predominantly capacitive detection as for predominantly resistive detection) [35].

Computational methods. A general boundary condition may always be reduced to superposition of appropriate coordinate transformations on an eigenpotential (e.g., $\Phi_{0}$ in eq 5). It is possible to obtain an analytical expression of the potential for the cubic boundaryvalue problem of eq 4 by the technique of variable separation and Fourier expansion [68]

$$
\Phi_{0}(x, y, z)=\frac{16}{\pi^{2}} \sum_{n=0}^{\infty} \sum_{m=0}^{\infty} \frac{\cos \left[\frac{\pi(2 n+1) z}{a}\right] \cos \left[\frac{\pi(2 m+1) y}{a}\right] \sinh \left[\frac{\pi(x-a / 2) \sqrt{(2 n+1)^{2}+(2 m+1)^{2}}}{a}\right]}{(2 n+1)(2 m+1) \sinh \left[\pi \sqrt{(2 n+1)^{2}+(2 m+1)^{2}}\right]}
$$

However, we have chosen a second method based on direct solution of the Laplace boundary problem by relaxation methods because (1) that method is not limited to a specific sel of boundary conditions and (2) it is much easier to extend the method to arbitrary ion trap geometry. For example, field inhomogeneity (such as that arising from finite spaces between the actual electrodes) may be treated easily with this method.

The excitation time-domain waveform may be specified as single-frequency excilation [1], frequencysweep (chirp) [32, 33], or SWIFT [34, 69]. The programs were written in Fortran 77 , compiled and executed on a CRAY Y-MP8/864 çompuler with 8 processors and 512 megabytes of high-speed memory at the Ohio Supercomputer Center. We chose the supercomputer for its high speed and precision and for its conveniently large internal memory space. To plot the desired potential, the data were transferred to a SPARCstation (Sun Microsystem Computer Corporation,
Mountain View, CA) through the OSU campus network and converted to a Felix data file format (Biosystem Technologies, San Diego, CA). Both contour and stacked plots may be generated within the Felix program. The trajectory data were transferred to a Macintosh IIsi [Apple Computer, Cupcrtino, C $\Lambda$ ] computer. They were displayed and printed with BASIC programs written by the authors. Listings of all relevant programs are available on request.

We used an apE software program specifically designed by The Ohio Supercomputer Center, Ohio Visualization Lab, to visualize the three-dimensional ion trajectory, excitation waveforms, and time-domain signal in real-time color video display. First, we convert the calculated data files (trajectory, excitation waveform, signal, etc.) into flux data format; spheres of different color and size represent ions of different position or mass-to-charge ratio. Second, the distribu- 
tion of spheres in a separately drawn cutaway ion trap at a particular time is recorded in an image file with resolution of $640 \times 484$. Finally, all image files are transferred onto a color videotape.

For the potential calculation, an array, $\Phi_{0}[i, j, k]$ of $101 \times 51 \times 51$ data words is reserved for the data: $0 \leq i \leq 100$ is the index for $\mathrm{x}$-position; $0 \leq j \leq 50$ and $0 \leq k \leq 50$ are the indices for $y$-and $z$-positions. Note that only one fourth of the eigenpotential data (101 $x$ $51 \times 51)$ need be generated, with corresponding reduction in computation time and storage space. The simplest relaxation method for computing the potential is described as follows. The potential values corresponding to the electrode at $x=-a / 2$ are set equal to 1 and those for the remaining boundaries are set to 0 . The potential values for the $n$th iteration, $\Phi_{0}^{n}(i, j, k)$, within the boundaries are calculated as the arithmetic average of the potential values at the six adjoining grid positions from the $(n-1)$ th iteration:

$$
\begin{aligned}
\Phi_{0}^{n}(i, j, k) \\
=\frac{1}{6}\left[\Phi_{0}^{n-1}(i-1, j, k)+\Phi_{0}^{n-1}(i+1, j, k)\right. \\
\quad+\Phi_{0}^{n-1}(i, j-1, k)+\Phi_{0}^{n-1}(i, j+1, k) \\
\left.\quad+\Phi_{0}^{n-1}(i, j, k-1)+\Phi_{0}^{n-1}(i, j, k+1)\right]
\end{aligned}
$$

The initial potential is $1 \mathrm{~V}$ for grid points on the plate, $\mathrm{x}=a / 2$, and $0 \mathrm{~V}$ for the rest of the grid points. Iteration stops when the maximum difference between the $n$th and $(n-1)$ th iteration for each point is less than a predetermined value. Typically, 10,000 iterations ( $\sim 6300 \mathrm{~s}$ processing time) were required to obtain potential values which differed by a maximum of $\leq 10^{-8}$ from the two consecutively preceding iterations. Although this potential computation is timeconsuming, it need be performed only once for a given trap geometry; the potential resulting from imposition of other voltages on each of the trap electrodes may then be obtained simply by rescaling the eigenpotential values.

During the trajectory simulation, the electric potential in the trap was computed at successive time increments from the eigenpotential for the cubic trap with appropriate boundary conditions [i.e., choice of what voltage was applied to which electrode(s)]. The electric field at a given ion position was computed as follows. First, move half of one grid spacing, $\Delta / 2$, away from the ion in the $+x,-x,+y,-y,+z$, and $-z$ directions, that is, $(x+\Delta / 2, y, z),(x-\Delta / 2, y, z)$, $(x, y+\Delta / 2, z),(x, y-\Delta / 2, z),(x, y, z+\Delta / 2)$, and $(x, y, z-\Delta / 2)$. The potential at each of those six locations is then obtained by linear interpolation from the potential values (labeled as $p_{1}$ to $p_{8}$ in Figure 1) at the eight points at the corners of the grid cube within which the desired location is found. For example, the

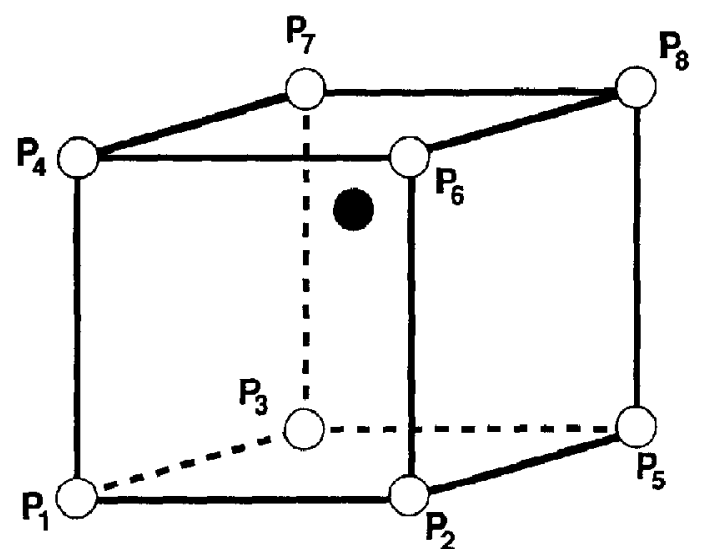

Figure 1. Schematic algorithm for computing the electric field at a given ion position. Start by moving half of one potential grid spacing away from the ion position, along the $+x,-x,+y,-y$, $+z$, and $-z$ positions. For each of those six positions, locate the eight nearest previously computed potential grid values (white circles at the corners of the cube surrounding a given solid circle). The potential at the solid circle is then obtained by linear interpolation of the potentials at the eight comers of the cube (see text).

potential at $(x+\Delta / 2, y, z)$ was calculated as follows:

$$
\begin{aligned}
V(x+ & \Delta / 2, y, z) \\
= & (1-t)(1-u)(1-v) P_{1}+t(1-u)(1-v) P_{2} \\
& +u(1-t)(1-v) P_{3}+v(1-t)(1-u) P_{4} \\
& +t u(1-v) P_{5}+t v(1-u) P_{6} \\
& +u v(1-t) P_{7}+t u v P_{8}
\end{aligned}
$$

in which:

$$
\begin{aligned}
& t=\frac{x+\Delta / 2}{\Delta}-\operatorname{int}\left(\frac{x+\Delta / 2}{\Delta}\right) \\
& u=\frac{y}{\Delta}-\operatorname{int}\left(\frac{y}{\Delta}\right) \\
& v=\frac{z}{\Delta}-\operatorname{int}\left(\frac{z}{\Delta}\right)
\end{aligned}
$$

Finally, the $\mathrm{x}-, \mathrm{y}^{-}$, and $\mathrm{z}$-components of the electric field, $\mathbf{E}=E_{x} \mathbf{i}+E_{y} \mathbf{j}+E_{z} \mathbf{k}$, at the ion position are obtained from the gradient of the potential, based on the potential values at $(x+\Delta / 2, y, z),(x-\Delta / 2, y, z)$, $(x, y+\Delta / 2, z),(x, y-\Delta / 2, z),(x, y, z+\Delta / 2)$, and $(x, y, z-\Delta / 2)$.

$$
\begin{aligned}
E_{x} & =\partial V(x, y, z) / \partial x \\
& \approx \frac{V(x+\Delta / 2, y, z)-V(x-\Delta / 2, y, z)}{\Delta} \\
E_{y} & =\partial V(x, y, z) / \partial y \\
& \approx \frac{V(x, y+\Delta / 2, z)-V(x, y-\Delta / 2, z)}{\Delta} \\
E_{z} & =\partial V(x, y, z) / \partial z \\
& \approx \frac{V(x, y, z+\Delta / 2)-V(x, y, z-\Delta / 2)}{\Delta}
\end{aligned}
$$


in which $\Delta$ is the distance between two adjacent grid points.

Finally, the Lorentz equations of ion motion (eq 8) were solved by the standard fourth-order Runge-Kutta numerical integration.

\section{Results and Discussion}

Electrostatic trapping potential in a cubic ICK ion trap. The most primitive approximation to the electrostatic field inside an ICR ion trap is no electrostatic field at all, because the Lorentz force resulting from the strong static magnetic field is usually at least an order of magnitude stronger than that due to the electrostatic trapping potential. This approximation is nearly realized in the screened trap [41], in which ions encounter a strong repulsive "trapping" force only as they almost reach the trapping electrodes. In the screened trap, the electrostatic trapping potential along the axial dimension is essentially a one-dimensional box and the potential in a plane perpendicular to the magnetic field is nearly zero.

Most of the present understanding of ion trapping in, for example, a cubic ICR ion trap is based on low order truncation of a Taylor expansion of the potential about the center of the trap. Near the center of the trap, the trapping potential may be well-approximated as quadrupolar:

$$
\Phi_{\text {trap }}=V_{T}\left[\gamma-\frac{\alpha}{2 a^{2}}\left(x^{2}+y^{2}-2 z^{2}\right)\right]
$$

in which $V_{T}$ is the trapping voltage applied to each of the two trapping electrodes (at $\mathrm{z}= \pm a / 2$ ) and the geometry parameters are $\gamma=1 / 3$ and $\alpha=2.77373$ for a cubic trap $[47,70]$. At this level of approximation, ion motions may be analyzed into three independent periodic modes, namely cyclotron, magnetron, and axial motions, each of whose frequency is independent of ion position in the trap [24]. The cyclotron frequency varies approximately as $1 /(\mathrm{m} / \mathrm{z})$, the trapping frequency as $1 / \sqrt{m / z}$, and the magnetron frequency (except near the critical mass [13]) is nearly independent of mass-to-charge ratio.

In an actual cubic trap, the trapping potential deviates from the quadrupolar approximation, and the cyclotron, magnetron, and axial frequencies vary with ion position in the trap. Figure 2 shows the electrostatic potential as a function of (axial) z-position along a line ( $x=0, y=0$ ) between the centers of the two endcap (or trapping) electrodes, as well as along two off-axis lines $(x=0.25 a, y=0$ and $x=0.45 a, y=0$ ). It is clear that the quadrupolar approximation to the actual cubic trap potential is quite good for ion positions near the z-axis and/or near the trap center $(x=y=z=0)$. However, the true cubic trap potential deviates increasingly from the quadrupolar approximation as one moves away from the trap center,

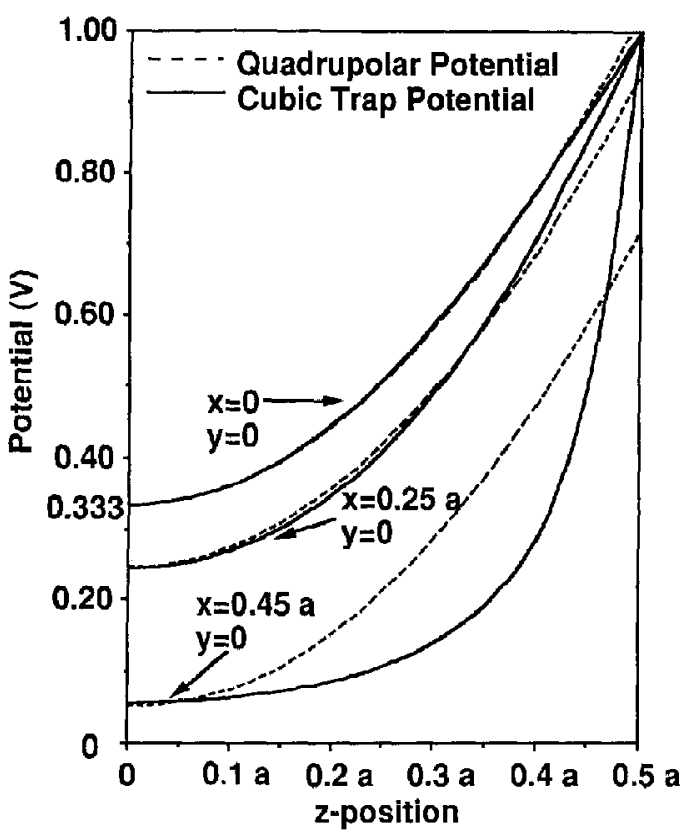

Figure 2. Electrostatic (trapping) potential as a function of $\mathrm{z}$ position at $(\mathrm{x}=0, \mathrm{y}=0),(\mathrm{x}=0.25 a, \mathrm{y}=0)$, and $(\mathrm{x}=0.45 a$, $y=0$ ), based on numerical computation for a cubic trap of side length, $a=1$ inch $(-)$ and corresponding quadrupolar $\left.(-)^{-}\right)$ potential. A $1 \mathrm{~V}$ direct current potential has been applied to each of the two trapping electrodes, with the other electrodes grounded.

particularly for radially outward displacement from the trap z-axis.

Deviation of the cubic trap potential from the quadrupolar potential is also evident from the equipotential contours shown in Figure 3, taken at the trap midplane $(z=0)$, and at planes midway $(z= \pm 0.25 a)$ or nearly as far as $(z= \pm 0.45 a)$ the trapping electrode. Note that the contours near the $z$-axis $(x=y=0)$ approach the perfect circles matching the quadrupolar approximation, whereas off-axis and (especially) $z \neq 0$ contours are increasingly distorted into rounded square curves.

Ion trajectory in the absence of time-varying electric field excitation. Figure 4 shows various representations of the trajectory of an unirradiated ion of $m / z 5000$. (We chose $m / z 5000$ so that the ratio of cyclotron frequency to magnetron frequency. would be small enough that magnetron, trapping, and cyclotron motions could be observed on a single time scale.) To illustrate the deviation of the real trap from the quadrupolar potential, we chose an initial ion position displaced from the trap center in both $\mathrm{x}$ - and $\mathrm{z}$-directions: $(x, y, z)=(0.4 a$, $0,0.25 a)$ were taken. As in a quadrupolar electrostatic trapping potential, ions in the cubic trap execute three basic motions: a fast radial circular ion cyclotron motion (the small loops in Figure 4, bottom left, and small 


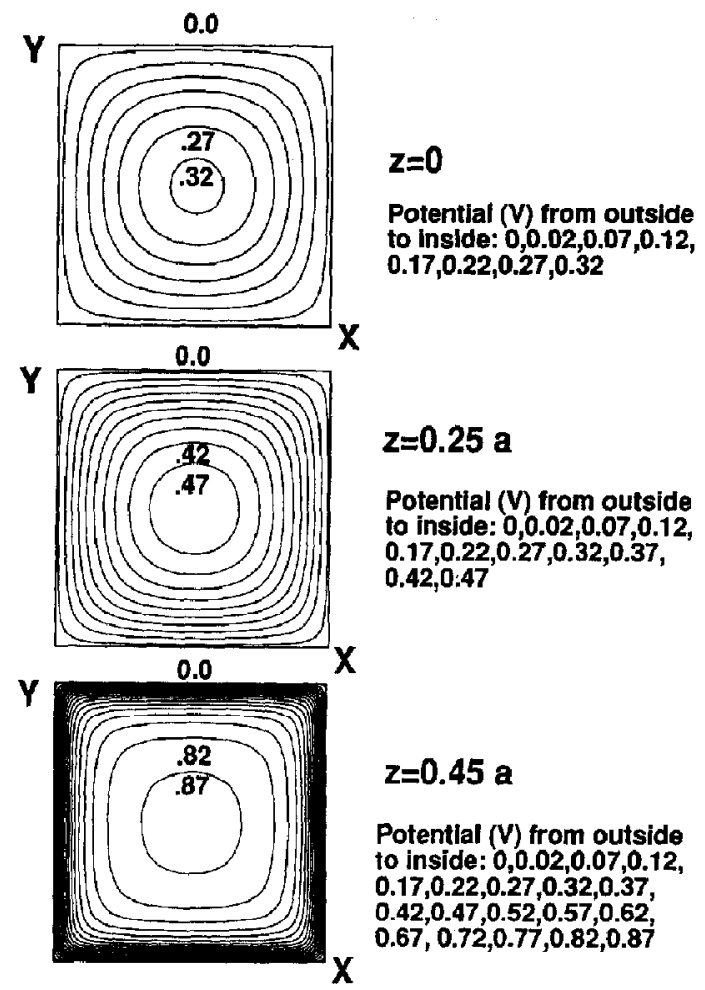

Figure 3. Equipotential contours of the electrostatic trapping potential in a cubic ICR ion trap of side length, $a=1$ inch, evaluated at the planes: $z=0, z=0.25 a$, and $z=0.45 a$. The four side electrodes are grounded and a potential of $1 \mathrm{~V}$ has been applied to each of the two trapping electrodes at $z= \pm 0,5 a$.
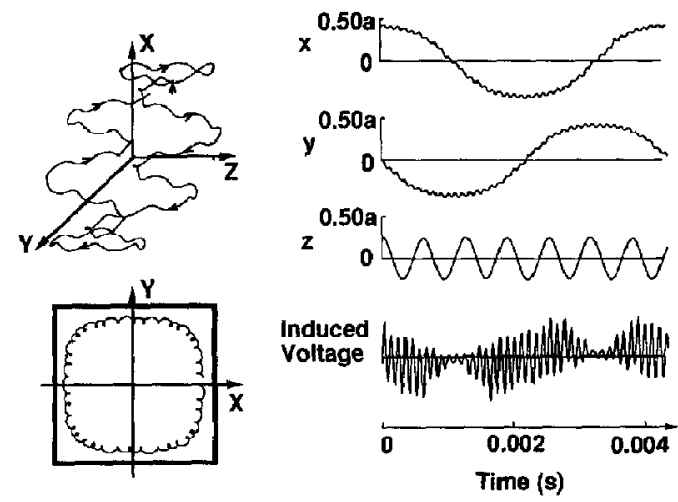

Figure 4. Various displays of the trajectory of ion of $\mathrm{m} / \mathrm{z} 5000$ with initial position $(0.40 a, 0.0,0.25 a)$ in a 1-inch cubic trap with $1 \mathrm{~V}$ applied to each of the two trapping electrodes. The initial ion velocity is zero. Upper left: three-dimensional ion spatial trajectory. Lower left: projection of the ion trajectory onto the xy-plane. Right: temporal variation of ion $x-, y^{-}$, and $z$-positions and differential current induced between two detector electrodes at $y= \pm a / 2 . B_{0}=3$ tesla. oscillations in Figure 4, top two right-hand graphs), a slow magnetron drift (large rounded square in Figure 4, bottom left, and slow oscillation in Figure 4, top two right-hand graphs), and an intermediate-frequency trapping oscillation along the z-direction (Figure 4, top left and second from bottom right).

Because most of the energy of magnetron motion is manifested as potential (rather than kinetic) energy, it is not surprising to find that magnetron motion is severely distorted by deviation of trapping potential from quadrupolar form. By comparing the projection of ion motion onto the xy-plane (Figure 4, bottom left) to Figure 3 (middle), it is seen that the magnetron motion follows the equipotential curve of the electrostatic potential of the cubic trap. The magnetron trajectory is thus approximately circular for an ion near the $z$-axis and distorts to a rounded square for an ion far from the z-axis. Less obviously, the time-variation of ion $\mathrm{x}$ - and $\mathrm{y}$-position (Figure 4, top right) shows that the ion moves faster when moving in a direction parallel to the nearest electrode and slower as the ion rounds the corners of the magnetron orbit. Thus, the differential current signal induced between two detection plates at $y= \pm a / 2$ (Figure 4, bottom right) is not simply a sum of sinusoids (see below).

Figure 5 shows how the radial component of electric field (Figure 5, left) and the corresponding magnetron frequency (Figure 5, right) vary with radial displacement from the trap $x$-axis. The average radial component of the electric field, $E_{r}$, is calculated by averaging over one cycle of average radius, $r$ (at increments of $1^{\circ}$ ), and the magnetron frequency is obtained from

$$
\omega_{-}=\frac{E_{r}}{r B}
$$

in which $B$ is the magnetic field induction. Figure 5 shows that the frequency of magnetron motion in a cubic trap decreases by around $40 \%$ in proceeding from an ion radius of $0.025 a$ (i.e., almost on the z-axis) to $0.475 a$ (i.e., near a side electrode), in good agreement with Dunbar and Chen's [49] prior two-dimensional numerical calculation. Because the magnetron motion and $z$-oscillation depend strongly on electrostatic field magnitude, the frequencies of both motions vary significantly with ion position in a cubic trap. Fortunately, in a typical FT/ICR/MS experiment, one excites and detects ion cyclotron motion, and even a large change in magnetron frequency will result in only a small shift in cyclotron frequency at mass-tocharge ratio values well below the critical mass-tocharge ratio at which the cyclotron frequency is equal to the magnetron frequency.

Ion trajectory in the presence of resonant electric field excitation. Figure 6 shows an example of an ion trajectory during single-frequency on-resonance dipolar excitation (10 $\mathrm{V}_{(\mathrm{p}-\mathrm{p})}$ amplitude) in a cubic trap [1 V applied to each trapping electrode; $m / z$ 1000, zero 

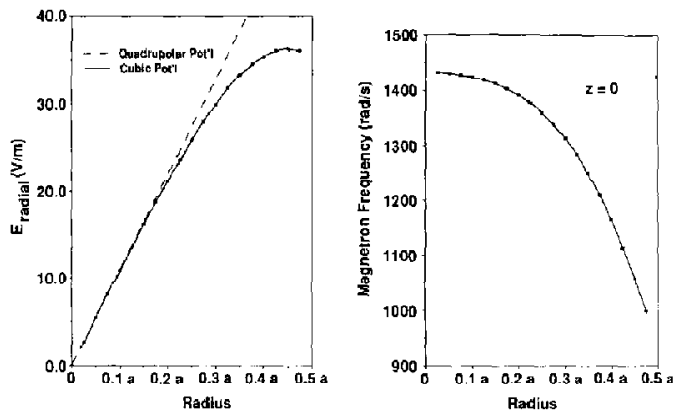

Figure 5. Radial component of electric field (left) and magnetron frequency (right) as a function of radial displacement from the trap $z$-axis, for a cubic trap with $1 \mathrm{~V}$ applied to each of the two trapping electrodes. The reported radial component of electric field and magnetron frequency are computed for $1^{\circ}$ increments and averaged over $360^{\circ}$.

initial kinetic energy, ion initial position $(0,0,0.25 a)]$. Because the ion is initially at rest on the z-axis, there is no magnetron motion, and the three-dimensional trajectory (Figure 6, top) is the sum of a linear constantamplitude oscillation along the z-direction (because

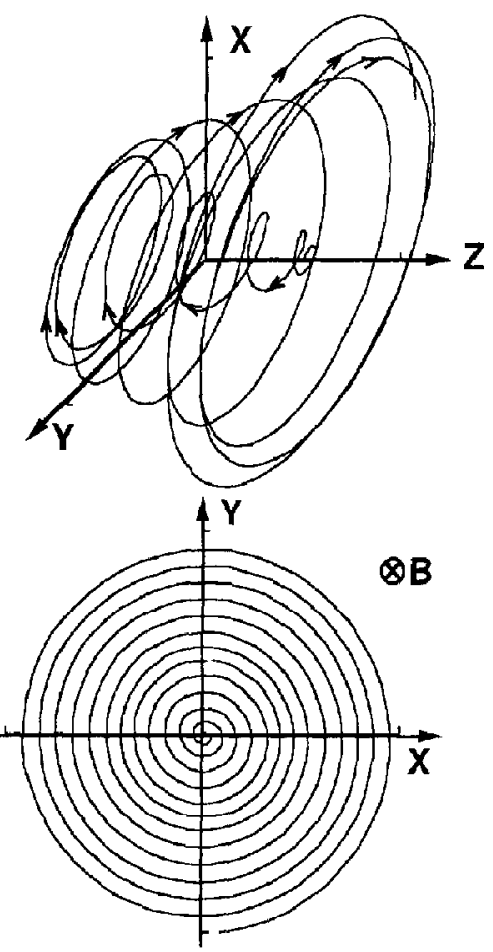

Figure 6. Ion trajectory during on-resonance single-frequency excitation $\left[10 \mathrm{~V}_{(\mathrm{p}-\mathrm{p})}\right]$ ] of an ion of $m / z 1000$, for ion initial position, $(0,0,0.25 a)$ in a cubic trap at 3.0 tesla ( $1 \mathrm{~V}$ direct current applied to each of the two trapping electrodes). Left: threedimensional display. Right: projection of the ion trajectory onto the $z=0$ plane through the center of the trap. the ion is initially displaced from the center of the trap along the $z$-axis) and a cyclotron motion of increasing cyclotron radius. The ion is ejected (i.e., reaches a cyclotron radius equal to the trap radius) after excitation for $2.72 \times 10^{-4} \mathrm{~s}$.

Projection of the trajectory onto the xy-plane (Figure 6 , bottom) shows that the ion cyclotron radius increases slightly nonlinearly with time due to spatial inhomogeneity of the RF excitation electric field. Specifically, the excitation field amplitude varies radially at any given axial ( $z$-) position. Moreover, an ion is subjected to a higher excitation field at the center of the trap than at a large z-displacement; thus, because the ion z-oscillation amplitude increases with increasing radius, even the time-average (over one trapping oscillation cycle) electric excitation field amplitude additionally varies with radius due to increased $z$ oscillation amplitude with increasing radius.

In response to spatially uniform resonant excitation in a purely quadrupolar trapping potential, ion cyclotron radius increases linearly with time, and ion kinetic energy increases quadratically with time. Figure 7 shows that an ion in a cubic trap behaves quite similarly, although the rate of increase in cyclotron radius is $\sim 30 \%$ less [47] than for the same excitation voltage applied to infinitely extended flat electrodes.

Ion trajectory in the presence of off-resonance electric field excitation. The trajectory of an ion during off-resonance excitation (namely, at a frequency $5 \%$ less than the ion cyclotron frequency) is shown in Figure 8 under otherwise identical conditions to those in Figures 6 and 7. (The time scale has been extended in Figure 8 relative to Figure 7 to make the $x$ - and $y$-amplitude ascillations easier to see.) An obvious feature of the new trajectory is the oscillation in cyclotron radius (top two graphs in Figure 8). Such off-resonance excitation has previously been analyzed in the absence of trapping potential [71], and by a rotating-frame treatment for a quadrupolar trapping potential [72], and offers a particularly attractive method for low-energy collision-induced dissociation ICR experiments [73].

Ion trajectory in the presence of collisions with/without quadrupolar excitation potential. In an ICR experiment, ions are confined radially due to the strong applied static magnetic field. To trap the ions along the $z$ direction, a positive potential is applied to each of the opposed pair of trapping electrodes to keep the positively charged ions (negative trapping potential for negatively charged ions) from escaping. Thus, at the center of the trap, the trapping potential is at a minimum along the $\mathrm{z}$-direction; however, it is at a maximum in the perpendicular direction (i.e., in the $x y$ plane). As a consequence, as shown in Figure 9 (left), ions are confined along an equipotential line if there is no energy loss mechanism; however, if an energy loss 

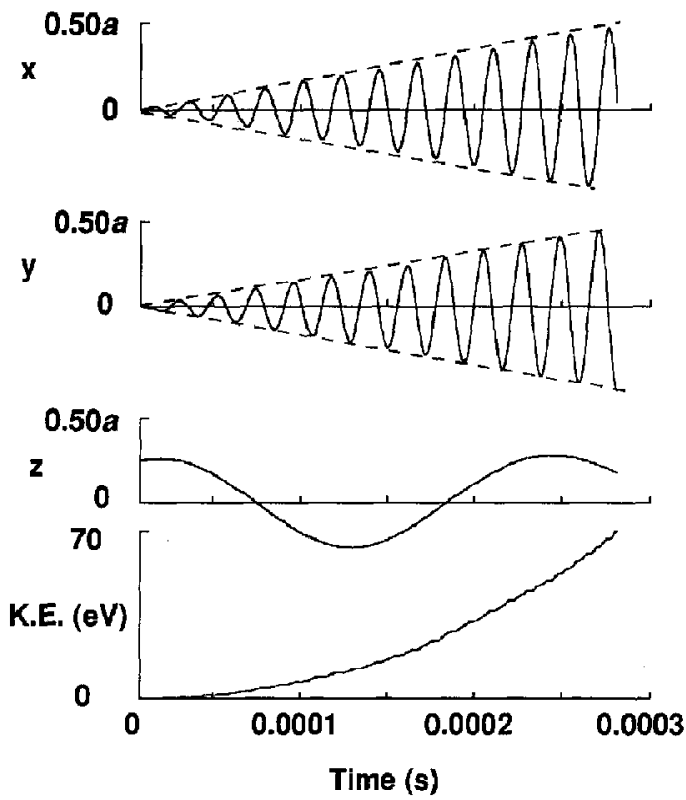

Figure 7. Temporal variation of ion $x-, y$-, and z-position components and total xy-kinetic energy (K.E.) for the ion of Figure 6. Note the linear increase in ion radial displacement and the quadratic increase in ion $x y$-kinetic energy during excitation, as well as the sinusoidal $z$-oscillation (trapping motion) independent of excitation.

mechanism such as frictional damping (as a model for ion-neutral collisions [51, 64]) is present, then the radius of ion cyclotron motion and the amplitude of z-oscillation decrease exponentially, whereas the magnetron radius increases exponentially at a slower rate. Thus, ion cyclotron and trapping motions rapidly damp to low amplitude, whereas the magnetron radius eventually increases until ions collide with the excitation or receiver electrodes and disappear, as shown in Figure 9 (middle).

Several ion cooling techniques $[74,75]$ have been proposed to eliminate the above ion loss mechanism. A quadrupolar excitation potential at frequency, $\omega_{c}$, converts magnetron motion to cyclotron motion $[37,76]$. Because the cyclotron radius decreases faster than the magnetron radius increases as a result of ion-neutral collisions, the net effect is that ions all eventually relax to the center of the trap (Figure 9, right). The quadrupolar excitation/collisional cooling technique offers the following advantages: (1) improved ICR peak shape and detection sensitivity (factor of 100 in some cases) [77]; (2) improved mass resolving power (up to factor of 500) [77]; (3) improved ion remeasurement efficiency (up to 99\%) for additional increase in signalto-noise ratio and /or multiple experiments with a single trapped-ion packet [78]; (4) extended trapping period to allow for thousands of ion-neutral collisions to release excess ion internal electronic or vibrational excitation energy [74]; (5) improved transfer efficiency
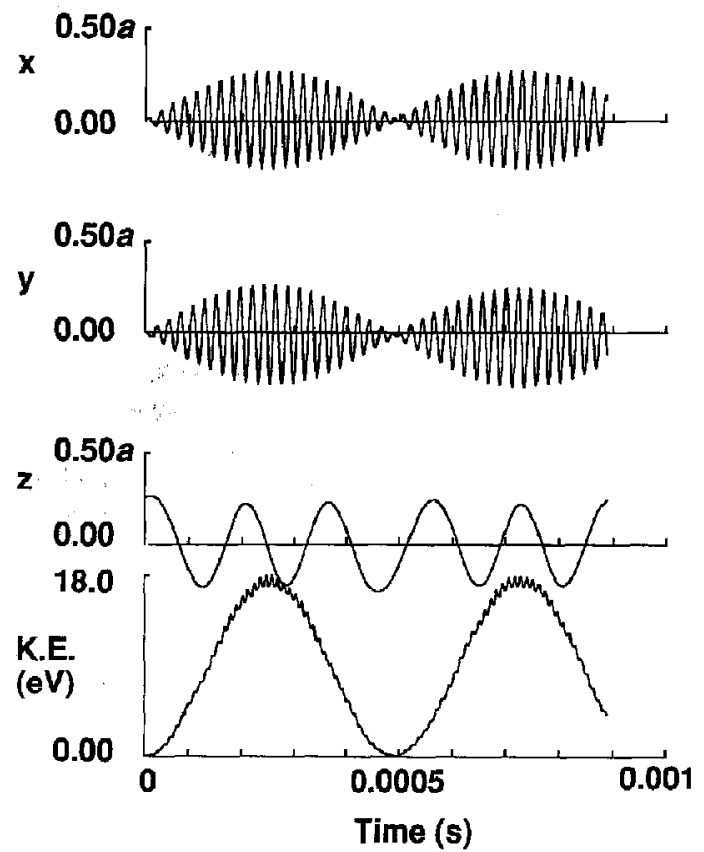

Figure 8. Temporal variation of ion $x-, y$-, and $z$-position components and total $x y$-kinetic energy (K.E.) during off-resonance single-frequency excitation (excitation frequency $=43548 \mathrm{~Hz}$, which is $\sim 5 \%$ lower than the reduced ion cyclotron frequency, $v_{+}$). Note the oscillatory ion radial displacement and oscillatory $x y$-kinetic energy.

to move ions through a narrow conductance limit [77]; (6) reduced z-ejection from excitation at $2 \omega_{z}$ and $\omega_{+} \pm$ $2 \omega_{z}$, etc. These features may now be examined by use of the present trajcctory simulations, as will be described in detail in a subsequent article.
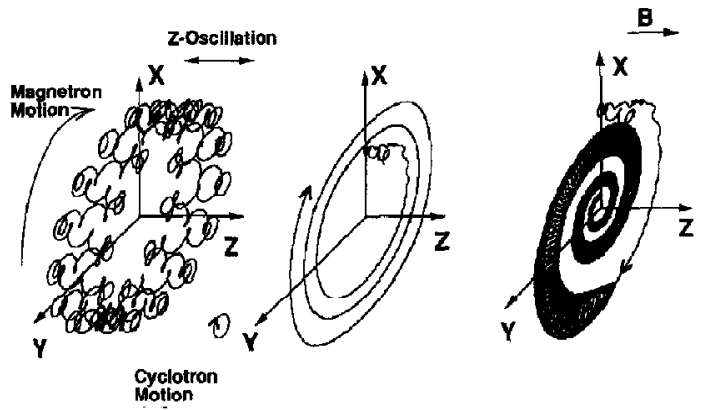

Figure 9. Ion trajectories for an ion of $m / z$ 1000; initial position $(0.3 a, 0,0.1 a)$; initial kinetic energy, $0.065 \mathrm{eV}$ (i.e., average kinetic energy equivalent to a temperature of $500 \mathrm{~K}$ ); trapping voltage, 2 $\checkmark$. Left: no collisional damping; no quadrupolar excitation. Middle: collisional damping with exponential damping constant of $10,000 \mathrm{~s}^{-1}$; no quadrupolar excitation. Right: as in Middle, for $0.001 \mathrm{~s}$ (see arrow), at which time a quadrupolar excitation potential of amplitude, $2 \mathrm{~V}_{(p-p)}$ and frequency, $\omega_{c}$ is applied. Note the conversion of magnetron motion to cyclotron motion, accompanied by rapid collisional damping of the cyclutron motion which relaxes ions to the center of the trap (sce text). 


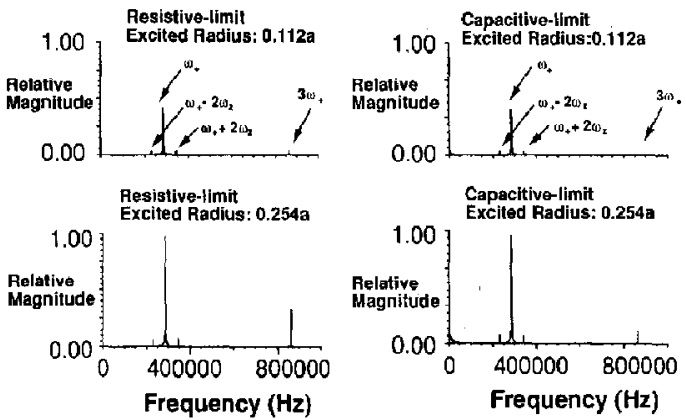

Figure 10. Frequency-domain FT/ICR spectra for ions of $m / z$ 5000 at 3.0 tesla, obtained by discrete Fourier transformation of the voltage signal derived from the induced differential current between two opposed detector electrodes of a cubic ICR ion trap (as in Figure 4), for an ion with initial position $(0,0,0.25 a)$ excited to an average cyclotron radius of $0.112 a$ (top) or $0.251 a$ (bottom), in the limit that the detection circuit is predominantly resistive (left) or predominantly capacitive (right). Ion initial $z$-velocity (directed toward the center of the trap) corresponded to a kinetic energy of $0.0385 \mathrm{eV}$. The ion trajectory was evaluated at time increments of $2.5 \times 10^{-7} \mathrm{~s}$ during both excitation and detection events. The dwell time (i,e, interval between two consecutive sampled time-domain data points) is $1.0 \times 10^{-5} \mathrm{~s}$. After excitation, ion cyclotron orbital radius is $0.12 a$ (top) and $0.25 a$ (bottom); ion kimetic energy is $4.74 \mathrm{eV}$ (top) and $17.8 \mathrm{eV}$ (bottom). There is a $29 \mathrm{~Hz}$ (downward) shift in ion cyclotron frequency and a pronounced increase in signal magnitude at harmonic and sideband frequencies as ions are excited to larger cyclotron radius (see text).

FT/ICR spectra. Figure 10 shows frequency-domain FI/ICR spectra obtained by discrete Fourier transformation of the voltage signal derived from the induced differential current between two opposed detector electrodes of a cubic ICR ion trap (as in Figure 4), for an ion with initial position $(0,0,0.25 a)$ excited to an average cyclotron radius of $0.112 a$ (top) or $0.254 a$ (bottom), in the limit that the detection circuit is predominantly resistive (left) or predominantly capacitive (right).

The signal component at the fundamental ICR frequency, $\omega_{+}$, clearly increases with increasing ICR radius, as seen in Figure 10 and quantitated in Table 2. Moreover, Figure 10 clearly reveals the presence of minor signal components at sideband $\left(\omega_{+} \pm 2 \omega_{z}\right)$ and odd-harmonic $\left(3 \omega_{+}, 5 \omega_{+}, 7 \omega_{+}\right.$etc.) frequencies. The radius-dependent magnitudes of the harmonic signals in fact furnish a means to measure ICR orbital radius directly in a cubic (or other shape) ICR ion trap [65, 79].

\section{Conclusions}

Here we present a simulation program that is capable of accurate calculation of the trajectory of an individual ion (in an empty ICR ion trap for any of several operating modes) and the corresponding frequencydomain spectrum of the voltage obtained from the time-derivative of the differential charge induced by that ion. This program provides a powerful tool for description and explanation (based on systematic variation of operating parameters) of various ion motions in an actual cubic ICR ion trap. For example, the single-ion response to any of various excitation modes recently analyzed in the limit of quadrupolar trapping potential and spatially uniform RF electric field [23] may now be accurately extended to the trapping and excitation electric potentials of an actual cubic trap. In particular, it should be possible to quantitate the " $\mathrm{z}$ ejection" phenomenon $[23,55,56,58-60]$, as will be reported in future work. Finally, extension of this program to incorporate the effect of space charge and collisions on the ion spatial distribution and detected ICR signtal is underway. A lisling of our program is available on request.

\section{Acknowledgments}

This work was supported by the National Science Foundation (CHE-90-21058), the Public Health Service (NIH GM-31683), The Ohio State University, and the National High Magnetic Field Laboratory at Florida State University. The authors thank The Ohio Supercomputer Center and Ohio Visualization Lab for providing the computing facilities.

\section{References}

1. Comisarow, M. B.; Marshall, A. G. Chem. Phys. Lett. 1974, 25, 282-283.

2. Freiser, B. S. Chemtracts-Analyt. \& Phys. Chem. 1989, 1. 65-109.

3. Ghaderi, S. Ceram. Transactions 1989, 5, 73-86.

4. Gord, J. R.; Freiser, B. S. Anal. Chim. Acta 1989, 225, 11-24.

5. Sharpe, P.; Richardson, D. E. Coord. Chem. Rev. 1989, 93 , 59-85.

6. Wanczek, K.-P. Int. J. Mass Spectrom. Ion Proc. 1989, 95, 1-38.

Table 2. Results for different initial ion position and excitation periods

\begin{tabular}{lccccc}
$\begin{array}{l}\text { Excitation } \\
\text { period(s) }\end{array}$ & $\begin{array}{l}\text { Initial } \\
\text { radius }\end{array}$ & $\begin{array}{c}\text { Initial } \\
\text { z-amplitude }\end{array}$ & $\begin{array}{c}\text { Final kinetic } \\
\text { energy (eV) }\end{array}$ & $\begin{array}{c}\text { Final } \\
\text { radius }\end{array}$ & $\begin{array}{c}\text { Detected signal } \\
\text { relative amplitude }\end{array}$ \\
\hline \hline $7.55 \times 10^{-5}$ & 0 & $0.25 a$ & $4 . / 4$ & $0.112 a$ & 4.313 \\
$1.50 \times 10^{-4}$ & 0 & $0.25 a$ & 17.77 & $0.254 a$ & 10.20 \\
$2.24 \times 10^{-4}$ & 0 & $0.25 a$ & 43.92 & $0.418 a$ & 19.92 \\
$7.55 \times 10^{-5}$ & 0 & 0 & 5.83 & $0.120 a$ & 5.333 \\
$1.50 \times 10^{-4}$ & 0 & 0 & 23.4 & $0.286 a$ & 12.98 \\
$2.24 \times 10^{-4}$ & 0 & 0 & 54.7 & $0.458 a$ & 26.70 \\
\hline
\end{tabular}


7. Wilkins, C. L.; Chowdhury, A. K,; Nuwaysir, L. M.; Coates, M. L. Mass Spectrom. Rev. 1989, 8, 67-92.

8. Freiser, B. S. In Bonding Energetics in Organometallic Compounds, vol. 428; Marks, T. J., Ed. American Chemical Society: Washington, DC, 1990; pp 55-69.

9. Laude, Jr., D. A.; Hogan, J. D. Terhnisches Messen 199n, 57, $155-159$.

10. Lubman, D. M., Ed.; Lasers in Mass Spectrometry. Oxford U. Press: New York, 1990.

11. Nibbering, N. M. M. Acc. Chem. Res. 1990, 23, 279-285.

12. Campana, J. E. In Proc. SPIE Applied Spectroscopy in Material Science; International Society for Optical Engineering: Bellingham, WA, 1991; pp 138-149.

13. Marshall, A. G.; Grosshans, P. B. Ant. Chem. 1991, 63, 215A-229A.

14. Nuwaysir, L. M; Wilkins, C. L. In Proc. SPIE Applied Spectroscopy in Material Science; International Society for Optical Engineering: Bellingham, WA, 1991; pp 112-123.

15. Eller, K,; Schwarz, H. Chem. Rev. 1991, 91, 1121-1177.

16. Dunbar, R. C. Mass Spectrom: Rev, 1992, 11, 309-339.

17. Jacoby, C. B.; Holliman, C. L.; Gross, M. L. In Mass Spectrometry in the Biological Sciences: A Tutorial; Gross, M. L., Ed. Kluwer Academic Publishers: Dordrecht, 1992; pp 93-116.

18. Köster, C.; Kahr, M. S.; Castoro, J. A.; Wilkins, C. L. Mass Spectrom. Rev. 1992, 11, 495-512.

19. Marshall, A. G; Schweikhard, L. Int. I. Mass Spectrom. Ion Proc. 1992, 118/119, 37-70.

20. Schweikhard, L.; Alber, G. M.; Marshall, A. G. Physica Scripta 1992, 46, 598-602.

21. Speir, J. P.; Gorman, G. S.; Amster, I. J. In Mass Spectrometry in the Biological Sciences: A Tutorial; Gross, M. L., Ed. Kluwer Academic Publishers: Dordrecht, 1992; pp 199-212.

22. Bucharan, M. V.; Hettich, R. L. Anal. Chem. 1993, 65, $245 \mathrm{~A}-259 \mathrm{~A}$.

23. Schweikhard, L; Marshall, A. G. J. Am. Soc. Mass Spectrom. 1993, 4, 433-452.

24. Brown, L. S.; Gabrielse, G. Rev. Mod. Phys. 1986, 58, $233-311$.

25. Gabrielse, G.; Haarsma, L.; Rolston, S. L. Int. I. Mass Spectrom. Ion Proc. 1989, 88, 319-332.

26. Kretzschmar, M. Physica Scripta 1992, 46, 544-554.

27. Kretzschmar, M. Eur. J. Phys. 1991, 12, 240-246.

28. Rempel, D. L.; Ledford, Jr., E. B.; Huang, S. K.; Gross, M. L. Anal. Chem. 1987, 59, 2527-2532.

29. Wineland, D. J.; Ekstrom, P.; Dehmelt, H. G. Phys. Rev. Lett. $1973,31,1279$.

30. Van Dyck, R. S. J.; Farnham, D. I.,; Shhwinberg, P. B. In NFFS 6/AMCO 9 Conference, 19-24 July; Bernkastel-Kues, Germany, 1992; pp 1-10.

31. Hearn, B. A.; Watson, C. H.; Baykut, G.; Eyler, J. R. Int. J. Mass Spectrom. Ion Prac. 1990, 95, 299-316.

32. Comisarow, M. B.; Marshall, A. G. Chem. Phys. Lett. 1974, 26, 489-490.

33. Marshall, A. G.; Roe, D. C. J. Chem. Phys. 1980, 73, 1581-1590.

34. Marshall, A. G.; Wang, T.-C. L.; Ricca, T. L. J. Amer. Chem. Soc. 1985, 107, 7893-7897.

35. Comisarow, M. B. J. Chem. Phys. 1978, 69, 4097-4104.

36. Limbach, P. A.; Grosshans, P. B.; Marshall, A. G. Anal. Chem. 1993, 65, 135-140.

37. Bollen, G.; Moore, R. B.; Savard, G.; Stolzenberg, H. Appl. Phys. 1990, 68, 4355-4374.

38. Yin, W. W.; Wang, M.; Marshall, A. G.; Ledford, Jr., E. B. J. Am. Soc. Mass Spectrom. 1992, 3, 188-197.

39. Nikolaev, E. N.; Gorshkov, M. V. Int. J. Mass Spectrom. lon Proc. 1985, 64, 115-125.

40. Kofel, P.; Allemann, M.; Kellerhals, H.; Wanczek, K.-P. Int. I. Mass Spectrom. Ion Prac. 1986, 74, 1-12.

41. Wang, M.; Marshall, A. G. Anal. Chem. 1989, 61, 1288-1293.

42. Wang, M.; Marshail, A. G. Anal. Chem. 1990, 62, 515-520.
43. Beu, S. C.; Laude, Jr., D. A. Anal. Chem. 1992, 64, 177-180.

44. Beu, Si C.; Laude, Jr., D. A. Int. J. Mass Spectrom. Ion Proc. 1992, 1i2, 215-230.

45. Comisarow, M. B. Int. J. Mass Spectrom. Ion Phys. 1981, 37. 251-257.

46. Sharp, T. F.; Fyler, J. R.; I.i, E. Int. I. Mass Spectrom. Ion Phys. 1972, 9, 421-439.

47. Grosshans, P. B.; Marshall, A. G. Int. I. Mass Spectrom. Ion Proc. 1990, 100, 347-379.

48. Lee, S. H.; Wanczek, K.-P.; Hartmann, H. Adv. Mass Spectrom. 1980, 8B, 1645-1647.

49. Dunbar, R. C.; Chen, J. H.; Hays, J. D. Int. J. Mass Spectrom. Ion Phys. 1984, 57, 39-56.

50. Hanson, C. D.; Castro, M. E.; Kerley, E. L.; Russell, D. H, Anal. Chem. 1990, 62, 520-526.

51. Wobschall, D.; Graham, Jr., J. R.; Malone, D. P. Phys. Rev. 1963, 131, 1565-1571.

52. Comisarow, M. B. J. Chem. Phys. 1971, 55, 205-217.

53. Guan, S. J. Am. Soc. Mass Spectrom. 1991, 2, 483-486.

54. Grosshans, P. B.; Marshall, A. G. Anal. Chem. 1991, 63, 2057-2061.

55. Huang, S. K,; Rempel, D. L.; Gross, M. L. Int. I. Mass Spectrom. Ion Proc. 1986, 72, 15-31.

56. Rempel, D. L.; Huang, S. K.; Gross, M. L. Int. J. Mass Spectrom. Ion Proc. 1986, 70, 163-184.

57. Allemann, M.; Kofel, P.; Kellerhals, H.; Wanczek, K.-P. Int. J. Mass Spectrom. Ion Proc. 1987, 75, 47-54.

58. van der Hart, W. J.; van de Guchte, W. J. Int. I. Mass Spectrom. Ion Proc. 1988, 82, 17-31.

59. van de Guchte, W. J.; van der Hart, W. J. Int. J. Mass Spectrom. Ion Proc. 1990, 95, 317-326.

60. Fujiwara, M.; Yamauchi, T.; Miura, K.; Inoue, M. J. Am. Soc. Mass Spectrom. 1992, 3, 847-852.

61. Mordehai, A. V.; Herion, J. D. Rapid Commun. Mass Spectrom. 1992, 6, 345-348.

62. Mitchell, D. W. Int. J. Mass Spectrom. Ion Proc. 1991, 107, 417-434.

63. Guan, S.; Gorshkov, M. V.; Alber, G. M.; Marshall, A. G. Phys. Rev. A 1993, 47, 2730-2737.

64. Beauchamp, J. L. J. Chern. Phys. 1967, 46, 1231-1243.

65. Grosshans, P. B.; Shields, P. J.; Marshall, A. G. J. Chem. Phys. 1991, 94, 5341-5352.

66. Dunbar, R. C. Int. J. Mass Spectrom, Ion Proc. 1984, 56, 1.

67. Shockley, W. J. Appl. Phys. 1938, 9, 635.

68. Jackson, J. D. Classical Electrodymamics; Wiley: New York, 1975; $\mathrm{p} 848$.

69. Marshall, A. G.; Wang, T.-C. L.; Chen, L.; Ricca, T. L. In American Chemical Society Symposium Series, vol. 359; Buchanan, M. V., Ed. American Chemical Society: Washington, DC, 1987; pp 21-33.

70. Byrne, J.; Farago, P. S. Proc. Phys. Soc. 1965, 86, 801-815

71. Beauchamp, J. L, Ann. Rev. Phys. Chem. 1971, 22, 527-561.

72. Wang, M.; Marshall, A. G. Int. J. Muss Spectrom. Ion Proc. 1990, 100, 323-346.

73. Gauthier, J. W.; Trautman, T. R.; Jacobson, D. B. Anal. Chim. Anta 1991, 246, 211-225.

74. Schweikhard, L.; Guan, S.; Marshall, A. G. Int. J. Mass Spectrom. Ion Proc. 1992, 120, 71-83.

75. Guan, S.; Xiang, X.; Marshall, A. G. Int. J. Mass Spectrom. Ion Proc. 1993, 124, 53-67.

76. Savard, G.; Becker, S.; Bollen, G.; Kluge, H.-J.; Moore, R. B.; Schweikhard, L,; Stolzenberg, $\mathrm{H}_{4}$; Wiess, U. Phys. Lett, A 1991, 158, 247-252.

77. Guan, S.; Wahl, M. C.; Wood, T. D.; Marshall, A. G. Anal. Chem. 1993, 65, 1753-1757.

78. Speir, J. P.; Gorman, G. S.; Pitsenberger, C. C.; Turner, C. A.; Wang, P. P.; Amster, I. J. Anal. Chem. 1993, 65, 1746-1752.

79. Grosshans, P. B.; Shields, P. J.; Marshall, A. G. J. Am. Chem. Soc. 1990, 112, 1275-1277. 